\title{
A NOTE ON WEAKLY MIXING FUNCTIONS
}

\author{
S. GLASNER
}

\begin{abstract}
Every almost periodic function is the uniform limit of polynomials of weakly mixing functions. If $\mathscr{B}$ and $\mathscr{F}$ are uniformly closed translation invariant algebras of purely weakly mixing functions and almost periodic functions respectively, and $\mathcal{Q}$ is the algebra generated by $\mathscr{B}$ and $\mathscr{F}$, then every weakly mixing function in $Q$ belongs to $\mathscr{B}$.
\end{abstract}

Let $\mathbf{Z}$ be the group of integers. It is well known that a bounded function $f$ on $\mathbf{Z}$ is almost periodic (a.p.) in the sense of Bohr iff there exist a compact Hausdorff space $X$, a homeomorphism $T$ of $X$ such that the flow $(X, T)$ is equicontinuous, a point $x_{0} \in X$ and a continuous function $\varphi$ on $X$ for which $f(n)=\varphi\left(T^{n} x_{0}\right)(n \in \mathbf{Z})$. We say that a function $f$ on $\mathbf{Z}$ is minimal (weakly mixing) if there exist a minimal (minimal and weakly mixing) flow $(X, T)$, $x_{0} \in X$, and a continuous function $\varphi$ on $X$ for which $f(n)=\varphi\left(T^{n} x_{0}\right)(n \in Z)$.

The set $\mathcal{E}$ of a.p. functions on $\mathbf{Z}$ is a uniformly closed, translation invariant algebra, a fact which corresponds to the existence of a universal minimal equicontinuous flow, namely the Bohr compactification of $\mathbf{Z}$. This is not the case with either the set of minimal functions [1], or the set of weakly mixing (w.m.) functions [5]. However, in the case of minimal functions, there exists a family of maximal algebras of minimal functions, all isomorphic to each other, whose union contains all minimal functions [2]. (Their intersection is the algebra of point distal functions [4].) This corresponds to the existence of $2^{c}$ idempotents in each of the minimal ideals of the semigroup $\beta \mathbf{Z}$, the Stone-Čech compactification of $\mathbf{Z}$, [6].

Let $u$ be one of these idempotents and let $\mathfrak{U}(u)$ be the corresponding maximal algebra of minimal functions. Inside $\mathfrak{U}(u)$ there are many maximal algebras of w.m. functions which are not necessarily isomorphic to each other [3]. (Their intersection is the algebra of purely weakly mixing functions.) The following are open questions. (i) Does the algebra generated by all minimal functions coincide with the algebra of all bounded functions on $\mathbf{Z}$ ? (ii) Does the algebra generated by all w.m. functions in $\mathfrak{A}(u)$ coincide with $\mathfrak{A}(u)$ ?

In this note we show that (1) the algebra generated by all w.m. functions in $\mathfrak{U}(u)$ contains $\mathcal{E}$, the algebra of a.p. functions, and (2) this result does not hold locally; i.e. there exists a uniformly closed, invariant algebra $\mathbb{Q} \subset \mathfrak{A}(u)$ (we call this simply an algebra) and two nontrivial subalgebras $\mathscr{B}$ and $\mathscr{F}$

Received by the editors August 19, 1978 and, in revised form, December 14, 1978.

AMS (MOS) subject classifications (1970). Primary 54H20.

Key words and phrases. Weakly mixing functions, almost periodic functions, purely weakly mixing functions. 
consisting of w.m. and a.p. functions respectively, and yet $\mathscr{F}$ is not contained in the algebra generated by all w.m. functions in $\mathbb{Q}$. In fact we show that for every purely weakly mixing (p.w.m.) algebra $\mathscr{B}$ and every a.p. algebra $\mathscr{F}$, every w.m. function in $\mathbb{Q}=\mathscr{B} \vee \mathscr{F}$ is contained in $\mathscr{B}$. We use the notations and terminology of [3].

THEOREM 1. Every almost periodic function is the uniform limit of polynomials of weakly mixing functions.

Proof. Let $(Y, T)$ be any minimal w.m. metric flow, $y_{0} \in Y$. Let $S=\{z \in$ C: $|z|=1\}$ be the unit circle and let $\sigma: Y \rightarrow S$ be a continuous function such that the flow on $X=Y \times S$ defined by $T(y, z)=(T y, \sigma(y) z)$ is minimal and w.m. [5]. Let $\lambda \in S$ be given and define a new flow on $X$ by $T^{\prime}(y, z)=$ $(T y, \lambda \sigma(y) z)$. By [3, Proposition 3.2] the orbit closure of $\left(y_{0}, 1\right)$ in $\left(X, T^{\prime}\right)$ is w.m. Let $\varphi: X \times X \rightarrow S$ be defined by $\varphi\left((y, z)\left(y^{\prime}, z^{\prime}\right)\right)=z^{-1} z^{\prime}$. Then $\varphi$ is a homomorphism of the pointed flow $\left(X, T,\left(y_{0}, 1\right)\right) \vee\left(X, T^{\prime},\left(y_{0}, 1\right)\right)$ into the pointed flow $\left(S, T_{\lambda}, 1\right)$ where $T_{\lambda}$ is the multiplication by $\lambda$.

Let $\mathbb{Q}_{1}$ and $\mathbb{Q}_{2}$ be the algebras corresponding to the pointed flows $\left(X, T,\left(y_{0}, 1\right)\right)$ and $\left(X, T^{\prime},\left(y_{0}, 1\right)\right)$ respectively. Then $\mathbb{Q}_{1}$, and $\mathbb{Q}_{2}$ are w.m. and the functions $\chi_{\lambda}(n)=\lambda^{n}$ is in the algebra $\mathscr{Q}_{1} \vee Q_{2}$. Since the function $\left\{\chi_{\lambda}\right.$ : $\lambda \in S\}$ span $\mathcal{E}$, the proof is completed.

THEOREM 2. Let $\left(Y, y_{0}\right)$ be a minimal p.w.m. pointed flow and let $\left(Z, z_{0}\right)$ be a minimal equicontinuous pointed flow. Put $\left(X, x_{0}\right)=\left(Y \times Z,\left(y_{0}, z_{0}\right)\right)$ and let $X \stackrel{\pi}{\rightarrow} Y$ be the projection. Suppose $\left(X, x_{0}\right) \stackrel{\varphi}{\rightarrow}\left(W, w_{0}\right)$ is a homomorphism where $\left(W, w_{0}\right)$ is w.m. Then there exists a homomorphism $\psi$ such that the diagram

$$
\begin{array}{cl}
\left(X, x_{0}\right) & \stackrel{\pi}{\rightarrow} \\
\varphi \downarrow & \swarrow \psi \\
\left(W, w_{0}\right) &
\end{array}
$$

is commutative.

Proof. ${ }^{1}$ Let $\mathscr{B}, \mathscr{W}$ and $\mathscr{F}$ be the algebras corresponding to the pointed flows $\left(Y, y_{0}\right),\left(W, w_{0}\right)$ and $\left(Z, z_{0}\right)$ respectively. We want to show that $\mathscr{W} \subset \mathscr{B}$ or equivalently that $\mathscr{B} \vee W \subset \mathscr{B}$. This will follow if we show that for every $p, q \in M(M$ a minimal ideal in $\beta Z) p|\mathscr{B}=q| \mathscr{B}$ implies $p|\mathscr{B} \vee W=q| \mathscr{B}$ $\vee \mathscr{W}$. Now since $\mathscr{B}$ is p.w.m, $\mathscr{B} \vee \mathscr{W}$ is a w.m. algebra and therefore disjoint from the almost periodic algebra $\mathscr{F}$. Thus, there exists an $r \in M$ with

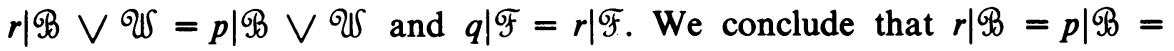
$q \mid \mathscr{B}$ and $r|\mathscr{B} \vee \mathscr{F}=q| \mathscr{B} \vee \mathscr{F}$. By assumption $\mathscr{B} \vee \mathscr{W} \subset \mathscr{B} \vee \mathscr{F}$ and consequently $p|\mathscr{B} \vee W=r| \mathscr{B} \vee W=q \mid \Re \vee \mho$

'I wish to thank the referee for suggesting this "algebraic" proof which is more transparent than my original proof. 


\section{REFERENCES}

1. H. Furstenberg, Disjointness in ergodic theory, minimal sets and a problem in Diophantine approximation, Math. Systems Theory 1 (1967), 1-49.

2. R. Ellis, Lectures on topological dynamics, Benjamin, New York, 1969.

3. R. Ellis and S. Glasner, Pure weak mixing, Trans. Amer. Math. Soc. 243 (1978), 135-146.

4. A. Knapp, Functions behaving like almost automorphic functions, Topological Dynamics, (Symposium, Colorado State Univ., Ft. Collins, Colo., 1967), Benjamin, New York, 1968, pp. 299-317.

5. R. Peleg, Some extensions of weakly mixing flows, Israel J. Math. 9 (1971), 330-336.

6. W. A. Veech, Private communication.

Department of Mathematics, Tel-Aviv University, Tel-Aviv, Israel 